\title{
La formación de profesionales reflexivos y la práctica de Enfermería
}

Mtra. Laura Morán Peña *

* Profesora de Carrera Titular B de TC de la ENEO - UNAM. Coordinadora del Programa de Maestría en Enfermería.

En este ensayo se plantea la necesidad de promover en los estudiantes de Enfermería el pensamiento reflexivo ya que como futuros profesionales de la Enfermería requerirán de habilidades de pensamiento crítico, en virtud de que la práctica de Enfermería a la que se enfrentarán implica sistemas de atención a la salud en los que los tipos de toma de decisiones, la complejidad de las necesidades de los pacientes y la cantidad y diversidad de información que utiliza se realiza, siempre, en situaciones de incertidumbre, de inestabilidad,con carácter único y con conflictos de valores. Se resalta la posibilidad de que una enseñanza diferente del proceso de atención de Enfermería bajo esta perspectiva pueda representar por sí misma la aplicación del pensamiento reflexivo.

Se concluye planteando algunas estrategias que deberán promover los docentes de Enfermería en los estudiantes para que desarrollen el pensamiento crítico como generador de juicios y toma de decisiones. Las estrategias enfatizan el desarrollo de conocimientos específicos; el aprendizaje experiencial progresivo; las competencias generales y específicas de pensamiento crítico, una enseñanza diferente del proceso de atención de Enfermería que promueva el razonamiento clínico y la inferencia diagnóstica; las habilidades de pensamiento crítico y de disposición al mismo; así como la conformación de estándares intelectuales y profesionales propios de un profesionista universitario.

PALABRAS CLAVE: pensamiento crítico, formación reflexiva, práctica de enfermería, actuar reflexivo.

\section{Abstract}

In this essay, the need to foster the reflexive thinking among nursing students since, as future nursing professionals, they will require critical thinking skills as well as those disposition qualities to it considering the nursing practice is performed in unique situations of uncertainty, instability as well as value conflicts. It's important to point out that the possibility of a different sort of teaching of the nursing process under this perspective can represent the application of the reflexive thinking.

It concludes stating some strategies that nursing teachers will have to foster among students for them to develop critical thinking as a judgment creator as well making decision. The strategies point out the development of specific knowledge; the progressive and experiential learning; the general and specific competences of critical thinking; a different approach of teaching the nursing process that foster clinical reasoning and the inferential diagnosis. The skills to set intellectual and professional standards which should be a characteristic of professional nurses.

Key words: critical thinking, reflective education, nursing practice, behave reflective. 


\section{INTRODUCCIÓN}

Las profesiones se han hecho esenciales para el funcionamiento mismo de nuestra sociedad. Pero aunque somos totalmente dependientes de ellos, hay signos crecientes de crisis de confianza en las profesiones, y en algunos casos hay fracasos evidentes de la acción profesional.'

Por ello, uno de los principales retos de las universidades del nuevo siglo es la formación de profesionales que sean capaces de resolver los problemas a los que se enfrenta cotidianamente en su ejercicio profesional.

Los profesionales a menudo reflexionan sobre lo que hacen, a veces incluso mientras lo están haciendo. Es este proceso completo de reflexión desde la acción el que resulta central para el "arte" mediante el cual los profesionales algunas veces reaccionan bien en situaciones de incertidumbre, inestabilidad, carácter único y conflicto de valores. $^{2}$

Esto representa un gran reto para las universidades, ya que como centros formadores de recursos humanos, tienen la responsabilidad de formar al alumno para el arte de contender con aquello con lo que va a encontrar en la realidad que no necesariamente tiene que ver con lo que le es enseñado en las escuelas o cómo les es enseñado en las escuelas.

En tiempos recientes se ha venido conformando un movimiento de reformas educativas a nivel internacional que tiene que ver con el desarrollo de pensamiento complejo en los futuros profesionales. Esto indiscutiblemente tiene que ver tanto con procesos cognitivos como con procesos sociales de construcción del conocimiento.

Dentro de este marco, las instituciones formadoras de recursos de Enfermería también han entrado en dicho movimiento y han buscado a través de diversas estrategias promover el desarrollo de pensamiento crítico. Experiencias tales como la promoción del aprendizaje basado en problemas, los cursos o talleres de desarrollo de habilidades del pensamiento y los programas de alta exigencia académica ofertados a los alumnos en el pregrado, han intentando promover su desarrollo.

No sobra decir, la importancia que tiene el desarrollo del pensamiento reflexivo, si consideramos que las Enfermeras, dada la naturaleza de sus actividades profesionales, deben tomar decisiones complejas, adaptarse constantemente a nuevas situaciones y actualizar continuamente sus conocimientos y habilidades, y que el pensamiento crítico forma parte integral de todo ello. ${ }^{3}$

Por lo tanto, los planes de estudio en Enfermería de todas las universidades plantean invariablemente, en los perfiles académico-profesionales la aspiración a desarrollar Enfermeros reflexivos, que sepan resolver problemas, que tomen las mejores decisiones respecto a las prácticas del cuidado y que tengan un espíritu indagador.

No obstante lo anterior, continúan observándose experiencias de formación enciclopedista, donde el alumno es receptor pasivo, en donde poco tienen que ver los aprendizajes significativos que promuevan la construcción de conocimiento científico de una manera diferente.

Por ello cobra relevancia el abordar esta temática, ya que el que las Enfermeras tengan un actuar reflexivo en las prácticas clínicas o comunitarias depende en gran medida en el tipo de formación que desarrollaron durante su formación como estudiantes de Enfermería.

\section{EL PROCESO DE ATENCIÓN DE ENFERMERÍA COMO UNA VÍA PARA EL DESARROLLO DEL PENSAMIENTO CRÍtIICO}

Por lo señalado anteriormente enfocaré la atención al desarrollo del pensamiento crítico en estudiantes de Enfermería y su relación con la elaboración que éstos hacen cuando elaboran juicios clínicos en específico cuando aprenden sobre una herramienta metodológica básica para ofertar cuidados que es el Proceso de Atención de Enfermería.

En México, a mediados de los años setenta fue introducido en las escuelas de Enfermería el Proceso de Atención de Enfermería, por grupos de profesoras formadas en Colombia. En ese entonces, aunque se le visualizaba como una herramienta metodológica para mejorar la calidad de las prácticas del cuidado, paradójicamente, permaneció varios años como estrategia de enseñanza y sólo, hasta años más tarde, fue introducido en los servicios de salud.

Quizá el que se haya introducido primero en la enseñanza es uno de los factores que han determinado que todavía se le conciba más como una metodología de enseñanza de los estudiantes de Enfermería y, consecuentemente, como un criterio final de acreditación de las materias de Enfermería, que como una estrategia metodológica de aplicación concreta y directa a la práctica, que implica, en sí misma, la constante identificación de problemas, la elaboración de juicios y la toma de decisiones. 
Numerosos autores como Alfaro-Lefevre, Potter y Perry ${ }^{4}$, Kozier ${ }^{5}$ y Kataoka-Yahiro y Saylor ${ }^{6}$, han planteado la importancia que tiene el pensamiento crítico ligado a la aplicación del Proceso de Atención de Enfermería, ya que en cada una de sus etapas éste está fuertemente implicado, pues tanto en su diseño, como en la ejecución y en la evaluación, se requieren diversas habilidades de pensamiento crítico, así como de tener disposición al mismo.

Desde un punto de vista teórico, el pensamiento crítico ha sido definido por una gran cantidad de autores de diversas maneras. Ya desde 1955 Bandman y Bandman ubicaban la necesidad de contar con Enfermeras que desarrollaran pensamiento crítico como una vía para enfrentar los complejos problemas de salud. Para ellos el pensamiento crítico es utilizado para generar ideas, inferencias y argumentos para tratar de manera lógica los aspectos y controversias de atención a la salud. ${ }^{8}$

Por su parte Watson y Glaser ${ }^{9}$ en 1964, definieron el pensamiento crítico como la composición de actitudes, conocimientos y habilidades que incluyen:

- $\quad$ Actitudes de indagación que involucran la habilidad para reconocer la presencia de problemas, y una aceptación de la necesidad general para apoyar con evidencias lo que se afirma como verdadero,

- Conocimiento de la naturaleza de las inferencias válidas y abstracciones y generalizaciones en las cuales el peso o la precisión de distintos tipos de evidencias son lógicamente determinadas

- Habilidades para el empleo y la aplicación de las actitudes y conocimientos ya mencionados.

Para este caso retomaré la planteada por la American Philosophical Association que lo definió como un proceso de juicio autoregulatorio y con propósito que resulta de la interpretación, análisis, evaluación e inferencia; así como de explicación por las consideraciones evidenciales, conceptuales, metodológicas y de criterio o de contexto en las que se basa ese juicio. ${ }^{10}$ De dicha definición obtenida por consenso, los doctores Facione y Facione derivaron tres instrumentos, el primero para evaluar las habilidades de pensamiento crítico, el segundo para evaluar la disposición al pensamiento críti$\mathrm{co}$, y el tercero consistente en un instrumento para evaluar el razonamiento clínico en las ciencias de la salud. Los dos primeros se encuentran disponibles en español.

Así como es importante desarrollar las habilidades del pensamiento crítico tales como la interpretación, el análisis, la evaluación, la inferencia, la explicación y la autorregulación, también lo es el desarrollo de la disposición al pensamiento crítico ya que es la parte afectiva, es una motivación interna consistente para identificar problemas y tomar decisiones utilizando el pensamiento.

En ese sentido Facione, Facione y Giancarlo en 1996, ${ }^{11}$ señalaron los siguientes hábitos mentales como componentes de la disposición en un pensador crítico ideal:

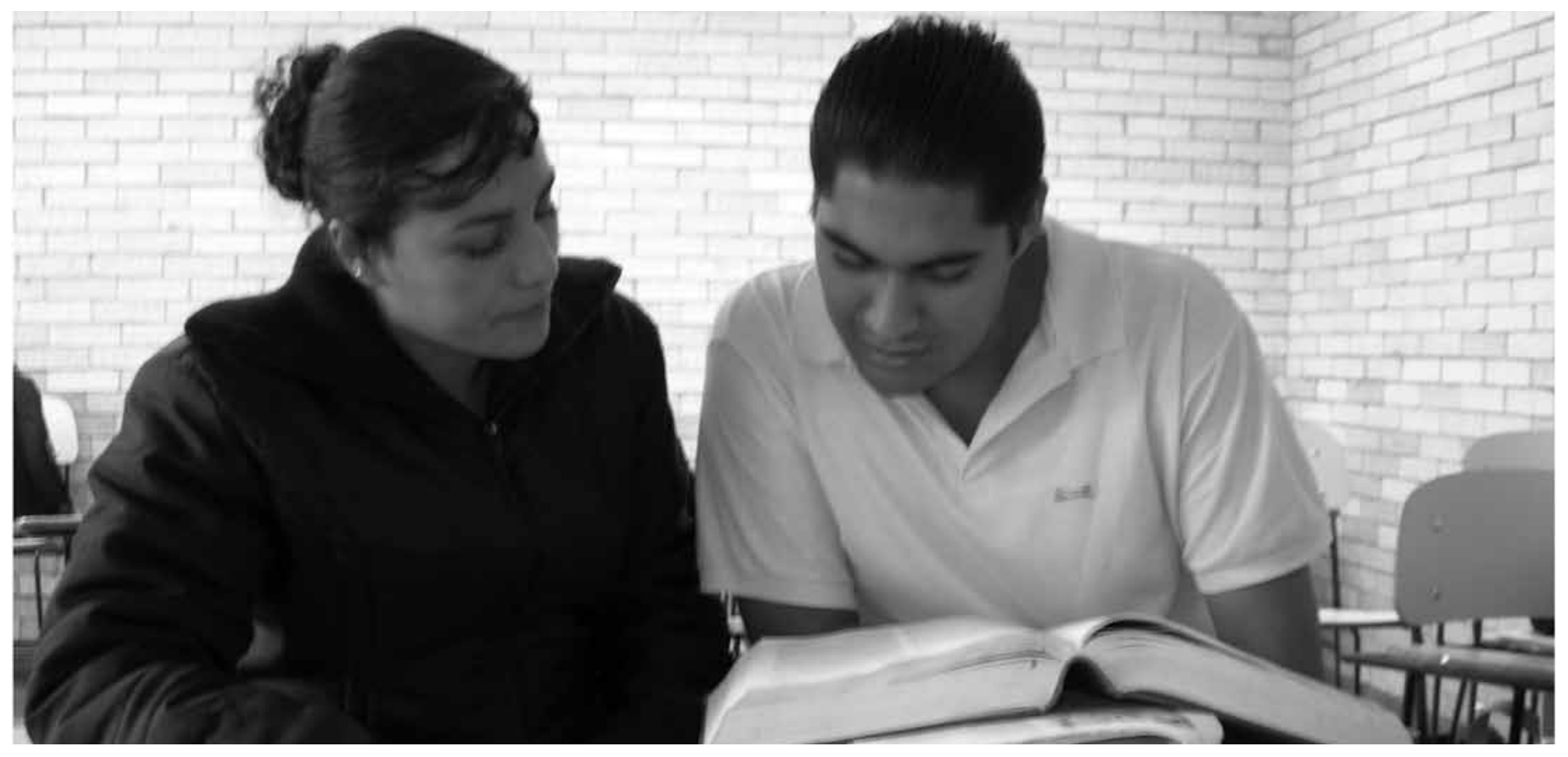


- Ser curioso.- deseo de adquirir conocimiento.

- Ser sistemático.- valorar la organización, la concentración y la diligencia en cualquier investigación.

- Ser analítico.- emplear la razón y la evidencia para resolver problemas.

- Buscar la verdad.- honestidad y objetividad con los hallazgos, incluso si no respaldan las propias creencias y las concepciones previas.

- Tener la mente abierta.- tolerancia con los puntos de vista divergentes.

- Autoconfianza de pensamiento crítico.- confianza en los poderes de razonamiento propios.

- Poseer madurez cognitiva.- prudencia al emitir, suspender o revisar juicios; reconocer que algunos problemas tienen más de una opción.

Sin duda coadyuvar a que los alumnos desarrollen dichos hábitos mentales es una tarea compleja para los docentes, pero indispensable si entendemos que educar es, ni más ni menos, que enseñar a pensar. $Y$ enseñar a pensar conlleva a promover en los estudiantes el juicio razonado, mismo que es desarrollado por ellos cuando se armonizan tres elementos: un ambiente de aprendizaje que nutre la disposición al pensamiento crítico como la aproximación preferida para definir y resolver problemas; la ejercitación de habilidades cognitivas en los estudiantes para ser aplicados en contextos siempre amplios y complejos que necesitan conocimiento profundo de los sujetos; y demandando la demostración de resultados así como de los procesos de juicios razonados en todas las tareas y pruebas de sus materias. ${ }^{12}$

\section{LA RELACIÓN DESARROLLO DE PENSAMIENTO CRÍTICO Y LA PRÁCTICA DE ENFERMERÍA}

Quizá lo que pueda acercarnos más a esta idea sea reconocer la relación pensamiento crítico y juicio enfermero. En ese sentido como ha sido señalado por Kataoka-Yahiro y Saylor, los resultados del pensamiento crítico es el juicio enfermero, es decir, el juicio clínico es el resultado de identificación y análisis de problemas relevantes de Enfermería en una diversidad de escenarios que implican situaciones de cuidado directo. ${ }^{13}$

Tañer señaló que los juicios clínicos incluyen: las decisiones sobre qué observar en las situaciones del paciente; decisiones inferenciales derivadas del significado de los datos observados; decisiones sobre las acciones que deberán ser tomadas y que darán el máximo beneficio para el paciente. Para ello, un estudiante de Enfermería deberá ir desarrollando de manera progresiva los siguientes aspec- tos: conocimiento específico de la disciplina, experiencia, competencias de pensamiento crítico, actitudes hacia el pensamiento crítico, así como estándares intelectuales y profesionales de pensamiento crítico.

Entonces los docentes en Enfermería tenemos un gran reto enfrente, pues como facilitadores del aprendizaje del estudiante tendremos que desarrollar estrategias que favorezcan las habilidades y destrezas mencionadas con anterioridad.

\section{CONCLUSIONES}

Derivadas del modelo planteado por Kataoka-Yahiro y Saylor ${ }^{14}$ se pueden sugerir algunas estrategias que posibilitarán el desarrollo del juicio clínico enfermero y por lo tanto posibilidades de desarrollar una práctica clínica o comunitaria de mayor calidad, es decir, un accionar reflexivo:

- Respecto a los conocimientos específicos a promover en los estudiantes de Enfermería no debemos olvidar que éstos implican tanto conocimientos como actitudes y habilidades. Podemos considerar que los conocimientos disciplinarios básicos proveerán datos para que los estudiantes elaboren diversos procesos de pensamiento crítico, por ejemplo, los datos obtenidos a través de la valoración de Enfermería, permitirán el razonamiento diagnóstico y la decisión sobre las intervenciones de Enfermería más apropiadas. En este sentido debemos favorecer que los estudiantes aprendan a acceder a los conocimientos científicos y humanísticos con los cuales, a través del pensamiento crítico, pueda elaborar los mejores juicios y toma de decisiones.

- $\quad$ Diversos autores como Tanner, Benner, Chesla y Gordon $^{15}$ han descrito la importancia del aprendizaje experiencial, ya que aprender el "know how" (saber cómo), permite tanto el reconocimiento de patrones como de respuestas intuitivas en el juicio experto de Enfermería. Y todo esto, como ha planteado Benner ${ }^{16}$, se logra de manera progresiva, ya que el entendimiento de situaciones complejas sólo se da a través de la experiencia con el análisis de situaciones similares y constrastantes. Por ello debemos promover que los estudiantes aprendan en los escenarios reales de la práctica clínica y comunitaria de manera progresiva.

- $\quad$ Respecto a las competencias que se deben promover en los estudiantes de Enfermería se pueden considerar: las competencias generales de pensamiento crítico a desarrollar en cualquier estudiante universitario (generación de hipótesis y resolución de problemas), 
las competencias de pensamiento crítico específicas para las situaciones clínicas (razonamiento diagnóstico, inferencias clínicas y toma de decisiones), y las competencias de pensamiento crítico específicas en la Enfermería ( las empleadas en el Proceso de Atención de Enfermería, en tanto que promueve un método racional y sistemático de planeación, ejecución y evaluación del cuidado de Enfermería a través de la utilización de procesos de pensamiento de alto orden). ${ }^{17}$

- $\quad$ Por ello es muy importante promover una enseñanza diferente del proceso de atención de Enfermería que promueva el razonamiento clínico y la inferencia diagnóstica a través de desarrollar en el estudiante habilidades para la adquisición de datos, la precisión diagnósti$\mathrm{ca}$, la toma de decisiones y la generación de hipótesis. Además de promover en los estudiantes actitudes hacia el pensamiento crítico tales como la confianza, independencia, responsabilidad, tomar riesgos, disciplina, perseverancia, creatividad, curiosidad, integridad y humildad.

- Respecto a los estándares para el pensamiento crítico como han sido señalado por Paul, ${ }^{18}$ están considerados los de carácter universal tales como claridad, precisión, especificidad, precisión, relevancia, plausibilidad, consistencia, lógica, profundidad y amplitud, entre otras cosas. En el caso de los estándares profesionales se encuentran los relativos a los criterios éticos para el juicio enfermero, los criterios para evaluación y la responsabilidad profesional. Habrá que promover el desarrollo de dichos estándares ya que es cada vez más común la evaluación de los profesionales a través de los procesos de certificación.

Sin duda alguna los contextos y las situaciones en las que se realizan las prácticas del cuidado de Enfermería son demasiado complejas y siempre se dan, como se señaló al principio, en situaciones de incertidumbre, inestabilidad, carácter único y conflicto de valores, por ello es necesario transformar la enseñanza, de tal forma que los estudiantes aprendan a aprender para la reflexión en la acción, para la reflexión sobre la acción y para la reflexión sobre la reflexión en y sobre la acción. Ello implica que los profesores desterremos estereotipos y que en principio seamos unos pensadores críticos, ya que eso permitirá ser modelo para los estudiantes. En concreto, tenemos un gran desafío, ya que para formar estudiantes que tengan un accionar reflexivo tenemos que ser profesores reflexivos y eso implica desarrollar las habilidades que queremos que nuestros propios estudiantes desarrollen.

\section{REFERENCIAS BIBLIOGRÁFICAS}

1 Schön D. La formación de profesionales reflexivos. Hacia un nuevo diseño de la enseñanza y el aprendizaje en las profesiones. Temas de Educación 28. Barcelona, 1992, Paidos. p. 18

2 Shön D. El profesional reflexivo. Como piensan los profesionales cuando actúan. Temas de Educación. 47, Barcelona, 1998, Paidos. p. 15-28

3 Alfaro-LeFevre R. El Pensamiento crítico en Enfermería. Un Enfoque práctico. Barcelona 1997, Masson. p. 4.

4 Potter,P.; Griffin,P. Fundamentos de Enfermería. $5^{\mathrm{a}}$ ed. St. Louis Missouri: Harcourt, 2002. p. 283-296.

5 Kozier B. Enfermería Fundamental. (Vols. 1-6), (4ª). Ed. Mc. Graw Hill-Interamericana. México, 1994. p.180-199.

6 Kataoka-Yahiro M and Saylor C. Critical thinking model for Nursing Judgment. In Journal of Nursing Education, April, USA, 1994, Vol. 33, 351- 360 .

7 Bandman E L \& Bandman B. Critical thinking in nursing. (2nd ed.) Norwalk: Appleton and Lange, USA, 1995. p.37

8 Rappsilber, Ch. et. al. Evaluación de habilidades de pensamiento crítico en estudiantes de Enfermería. Un estudio comparativo. Universidad Autónoma de Nuevo León, México, 1999. p. 5-24

9 Wattson $\mathrm{G}$ and Glaser E. Critical thinking appraisal manual. New York: Hartcourt Brace Jovanovich, 1980 p. 4-6.

10 Facione P A. Facione, N. California Critical Thinking Skills Test. Test. Manual Form A \& B. Milbrae, USA: California Academic Press, 1998. p. 2.

11 Facione PA, Facione NC, Giancarlo CA. Critical thinking disposition as a mesure of competent clinical clinical judgement: the development of the CCTDI. Journal of Nursing Education. USA, 1994, Vol. 33. p. 345-350.

12 Facione P A. Facione, N. Critical thinking as reasoned judgment. Teaching for and about critical thinking. In "The Critical Thinking Album" Disponible en: www.insightassessment. com/articles/html The California Academic Press, 2005. pp. $1-45$

13 Idem p. 6

14 Idem p. 6

15 Gordon M. Dianóstico Enfermero. Procesos y aplicación. MosbyDoyma, México, 1996. p. 132-166.

16 Benner P. Práctica progresiva en Enfermería. Manual de Comportamiento profesional. Serie: Enfermería y sociedad. Grijalbo, México, 1987. p. 41-65.

17 Kozier B, Erb G, Blais K. Fundamentos de Enfermería. Conceptos, proceso y práctica. $5^{\text {a }}$ ed. Vol.1. Barcelona, 1999, Interamericana Mc-Graw-Hill, 1999. p.177.

18 Paul R. Critical thinking: How to prepare students for a rapidly changing world. California: Foundation for critical thinking. USA, 1993. p. 24-26

\section{DIRECCIÓN PARA CORRESPONDENCIA}

Laura Morán Peña: eneolm@yahoo.com 